\title{
Respiratory decline in adult patients with Becker muscular dystrophy: A longitudinal study
}

\author{
Bram De Wel $^{\mathrm{a}, \mathrm{b}, 1}$, Sofie Willaert ${ }^{\mathrm{c}, 1}$, Aleksandra Nadaj-Pakleza ${ }^{\mathrm{d}}$, Anne-Catherine Aubé-Nathier ${ }^{\mathrm{e}}$, \\ Dries Testelmans ${ }^{\mathrm{f}}$, Bertien Buyse ${ }^{\mathrm{f}}$, Kristl G. Claeys ${ }^{\mathrm{a}, \mathrm{b}, *}$ \\ ${ }^{a}$ Department of Neurology, Neuromuscular Reference Centre, University Hospitals Leuven, Leuven, Belgium \\ ${ }^{\mathrm{b}}$ Laboratory for Muscle Diseases and Neuropathies, Department of Neurosciences, and Leuven Brain Institute, KU Leuven, Leuven, Belgium \\ ${ }^{\mathrm{c}}$ Faculty of Medicine, KU Leuven, Leuven, Belgium \\ ${ }^{\mathrm{d}}$ Department of Neurology, Neuromuscular Reference Centre Nord/Est/IdF, CHU Strasbourg, Strasbourg, France \\ ${ }^{\mathrm{e}}$ Department of Neurology, Neuromuscular Reference Centre AOC, CHU Angers, Angers, France \\ ${ }_{\mathrm{f}}^{\mathrm{f}}$ Department of Pulmonology, Leuven University Centre for Sleep and Wake Disorders, University Hospitals Leuven, Leuven, Belgium
}

Received 4 October 2020; received in revised form 13 December 2020; accepted 21 December 2020

Available online $\mathrm{xxx}$

\begin{abstract}
Becker muscular dystrophy (BMD) is a rare hereditary neuromuscular disease, caused by a genetic defect in the Duchenne Muscular Dystrophy $(D M D)$ gene. We studied the natural history of respiratory function and its affecting factors in 23 adult BMD patients. These important data are needed for (future) clinical trials in BMD but are largely lacking. Patients had a median age of 51 years (28-78y) and median follow-up duration of 14 years $(2-25 y)$. We analysed 190 pulmonary function measurements with a median interval of one year $(1-17 y)$ and measured a $1.00 \%$ decline of Forced Vital Capacity percent predicted (FVC\%pred) per year $(p=0.004)$. Loss of ambulation significantly increased the annual rate of FVC decline and was dependent of patient's body mass index (BMI; $p=0.015)$, with increases in BMI correlating with an even more rapid deterioration of FVC. A decline in Medical Research Council (MRC) sum score was significantly correlated with a decline in FVC $(p=0.003)$. We conclude that adult BMD patients experience a significant but mild respiratory decline. However, this decline is significantly more rapid and clinically relevant after loss of ambulation, which warrants a more vigilant follow-up of respiratory function in this subgroup.
\end{abstract}

(C) 2020 Elsevier B.V. All rights reserved.

Keywords: BMD; DMD-gene; FVC; spirometry; BMI; Natural history.

\section{Introduction}

Becker muscular dystrophy (BMD) is an X-linked neuromuscular disease, caused by a genetic defect in the Duchenne Muscular Dystrophy $(D M D)$ gene, leading to a deficient, less functional dystrophin protein. In contrast, DMD is caused by a complete absence of dystrophin protein and is accompanied by a more severe phenotype. In general, in-frame mutations lead to a BMD phenotype

\footnotetext{
* Corresponding author at: Department of Neurology, University Hospitals Leuven, Campus Gasthuisberg, Herestraat 49, 3000 Leuven, Belgium.

E-mail address: kristl.claeys@uzleuven.be (K.G. Claeys).

${ }^{1}$ These authors contributed equally to this work.
}

and out of frame mutations to DMD, although exceptions occur [1]. BMD is a rare disease with a prevalence of around 2.5/100.000 [2]. Patients with BMD are clinically characterized by progressive weakness and atrophy of the proximal limb muscles, with symptom onset usually in adolescence. However, BMD phenotypes vary notoriously from a DMD-like clinical progression with severe limb-girdle muscle weakness and cardio-respiratory complications to mild symptoms such as fatigue or muscle cramps.

Natural history studies in BMD are very rare and longitudinal studies exploring respiratory function in BMD patients are largely lacking [3,4]. In contrast, much more is known on cardiac complications in BMD, with dilated cardiomyopathy and/or conduction abnormalities as typical features [5]. 
Novel (genetic) therapies are being developed for DMD but might also be applicable in some BMD patients [6]. With these emerging potential treatments on the horizon, a thorough understanding of natural disease progression is important, as well as the availability of valid outcome measures. In DMD, forced vital capacity (FVC) has been proposed as a reliable outcome measure for respiratory function, suggesting that this parameter might also be relevant to follow-up BMD patients [7].

Here, we studied the rate of respiratory decline in a cohort of adult BMD patients and explored clinical variables associated with this deterioration.

\section{Patients and methods}

\subsection{BMD patients and data collection}

In this retrospective study, we included 23 adult $(\geq 18$ years) patients with genetically confirmed BMD: 14 patients were followed at the neuromuscular reference centre at University Hospitals Leuven (Leuven, Belgium) and 9 patients at the University Hospital Angers (Angers, France). Additional inclusion criteria were the availability of at least two spirometry measurements at different time points and measurable skeletal muscle weakness on the Medical Research Council (MRC) sum score ( $\leq 59 / 60$ points). The last additional criterion for inclusion was applied to increase phenotypic homogeneity.

We collected the following data from the patients' medical files: age at symptom onset, first symptom(s), ambulatory status, body mass index (BMI), presence of proximal and/or distal weakness in arms and legs, MRC sum score, contractures, scoliosis, scapular winging, calf hypertrophy, cause of death, genetic defect, family history, left ventricular ejection fraction (LVEF), presence and type of cardiomyopathy, cardiac conduction abnormalities, pacemaker implantation, history of heart transplantation, FVC, FVC percent predicted (FVC\%pred), forced expiratory volume in 1 s (FEV1), FEV1 percent predicted (FEV1\%pred), peak expiratory flow (PEF) and ventilatory support (Table 1).

We focussed mainly on FVC as the measure of respiratory function, since FVC was previously shown to be one of the most important parameters to monitor respiratory muscle function in the related muscle disease DMD [8]. The Ethics Committees approved this retrospective study.

\subsection{Methodology of FVC, MRC sum score and LVEF measurement}

Pulmonary function tests were performed with Jaeger ${ }^{\circledR}$ spirometers by a group of trained professionals at the pulmonology department. Thus, no bedside testing was done. All patients received the same instructions and were inquired for smoking behaviour. Only the best result of three consecutive measurements was noted. For measurements in sitting position, non-ambulatory patients were allowed to stay in their wheelchair and were positioned in front of the spirometer. Measurements in supine position in nonambulatory patients were performed after transferring them onto a gurney. Spirometers were volume-calibrated twice a day, biologically calibrated once a week and received standard maintenance once a year. Reference values of the Global Lung Function Initiative were used (GLI 2012) [9].

The MRC sum score (0-60 points) was measured as part of the standard of care by manually testing strength in six individual muscle groups at both sides: shoulder abduction, elbow flexion, wrist extension, hip flexion, knee extension and foot dorsiflexion [10].

The LVEF was calculated following the guidelines of the European Society of Cardiology and measured with $\mathrm{GE}^{\circledR}$ echocardiography devices by trained professionals [11]. Methodology of testing was similar in both centres.

\subsection{Statistical analysis}

Statistical analyses were performed using RStudio ${ }^{\circledR}$ Desktop (Open Source License, version 1.2.5001). All available patient data were used for analyses. Descriptive statistics are stated as percentages, median (range) or mean (standard deviation, SD) where appropriate. We used Linear mixed models (LMM; lme4 and lmerTest packages) to analyse longitudinal changes of lung function, with patient identification as a random effect to avoid pseudoreplication. These models contained age at measurement as a fixed effect, as well as a random slope for patients' age, to allow for differences in rate of pulmonary function decline between patients (reflecting disease heterogeneity). The LMM analysing the correlation of patient characteristics with FVC decline, contained each of the analysed variables as a fixed effect, as well as an interaction term between the amount of years since loss of ambulation and BMI. Multiple linear regression was used for the analysis of cross-sectional data (the correlation between the clinical neurological examination at the time of each patients' last visit and the FVC values at that time). All statistical models are detailed in Supplementary Table 1. Significance level was determined at $\alpha=0.05$.

\section{Results}

\subsection{Demographic, clinical, and genetic features of the adult BMD cohort (Table 1)}

The 23 adult BMD patients had a median age of 51 years (range 28-78y), a median age at symptom onset of 10 years (range 3-40y) and median follow-up duration at our hospitals of 14 years (range 2-25y). The 23 patients had 190 pulmonary function measurements and the median interval between these measurements was one year (range 117y). At the most recent clinical examination, all patients suffered from proximal weakness in the legs, 19 patients additionally experienced proximal weakness in the arms (83\%) and eight patients presented scapular winging (50\%, $8 / 16)$. Twelve patients showed calf hypertrophy (52\%). There was a significant positive correlation between the presence 
Table 1

Clinical, cardio-respiratory and genetic features of the BMD patient cohort.

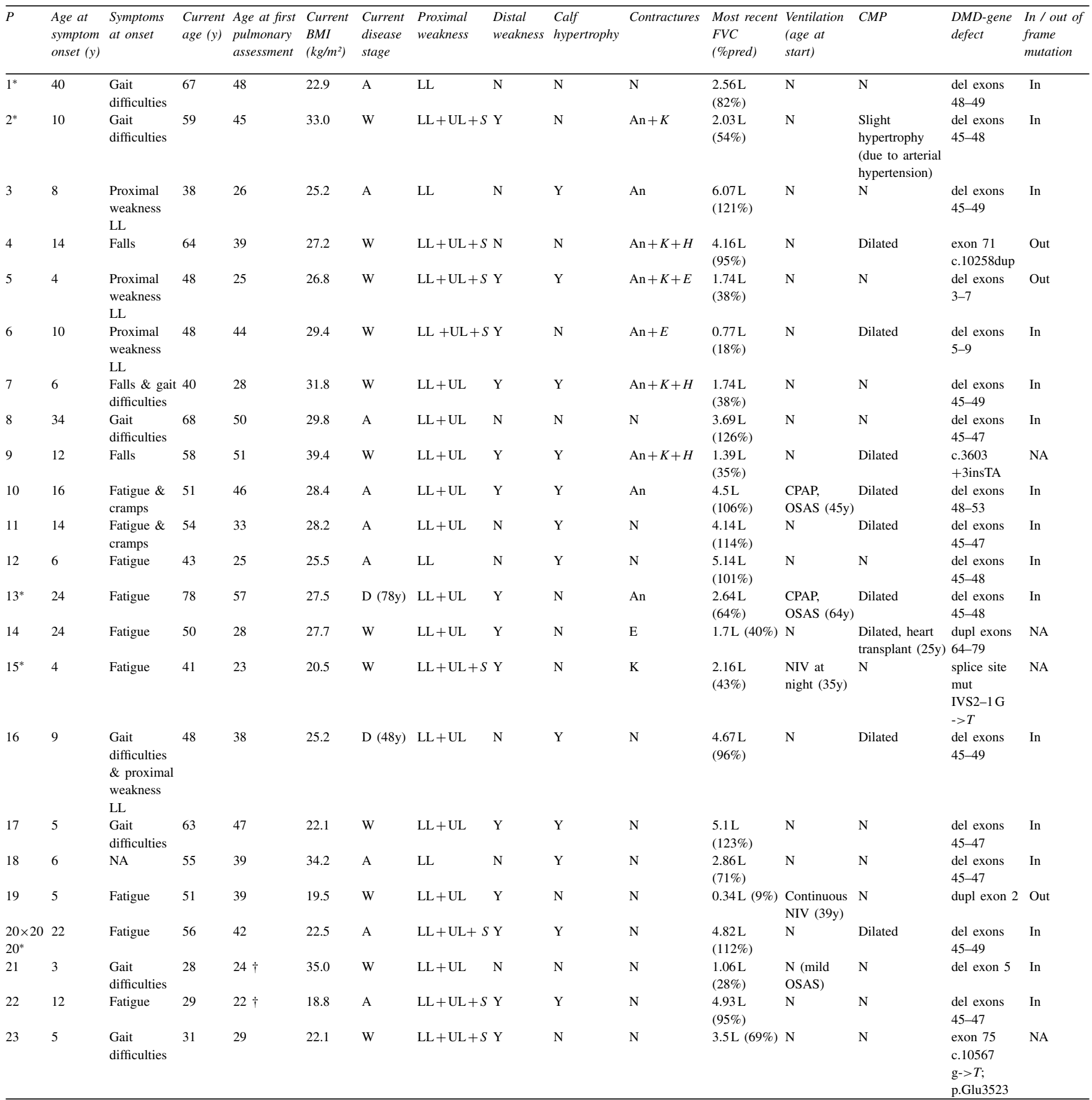

Becker Muscular Dystrophy (BMD), Patient (P), Body Mass Index (BMI), Forced Vital Capacity, (FVC), cardiomyopathy (CMP), percentage of predicted (\%pred), Duchenne Muscular Dystrophy (DMD), Yes (Y), No (N), Not Available (NA), Ambulatory (A), Wheelchair bound (W), Deceased (D), scapular winging (S), upper limbs (UL), lower limbs (LL), Ankles (An), Knees (K), Hips (H), Elbows (E), Non-invasive ventilation (NIV), Continuous positive airway pressure (CPAP), Obstructive sleep apnoea syndrome (OSAS), deletion (del), duplication (dupl), mutation (mut). Patients with active or past smoking behaviour are indicated with an asterisk $\left({ }^{*}\right)$. $\dagger$ P21 and P22 had pulmonary function tests during childhood (at ages 7/8/9/11/13y (P21) and age 12y (P22)) but these were within normal ranges and not included in the statistical analysis. The indicated age at first pulmonary assessment in the table for these patients was the age at first spirometry in adulthood. For all other patients no prior data of pulmonary function tests were available. 
Table 2

Cardiac complications and therapies in BMD patients.

\begin{tabular}{lll}
\hline$P$ & Cardiac complications (age) & Cardiac medication \\
\hline 2 & Hypertrophic CMP (36) \& RBBB (NA) & Beta-blocker, ACE-I, diuretic \\
4 & Dilated CMP (NA) & N \\
5 & Borderline low LVEF (39), Total AV block: pacemaker (47) & Beta-blocker, ACE-I \\
6 & Dilated CMP (NA) & N \\
8 & AV-block type 1 (50) & Beta-blocker, ARB \\
9 & Dilated CMP (NA), Total AV block: pacemaker (NA) & N \\
10 & Dilated CMP (47) & Beta-blocker, ARB, calcium antagonist \\
11 & Dilated CMP (53) & Beta-blocker, ACE-I \\
13 & Dilated CMP (56), total AV block: pacemaker (73) & Beta-blocker, ACE-I \\
14 & Dilated CMP (18), heart transplantation (25) & Beta-blocker, ARB \\
16 & Dilated CMP (42) & N \\
20 & Dilated CMP (42) & ACE-I \\
21 & RBBB (NA) & Beta-blocker, ACE-I \\
\hline
\end{tabular}

Only patients suffering from cardiac complications are mentioned in this table. Cardiomyopathy (CMP), Right Bundle Branch Block (RBBB), Not available (NA), Left Ventricular Ejection Fraction (LVEF), Atrioventricular (AV), Angiotensin Converting Enzyme Inhibitor (ACE-I), No (N), Angiotensin II receptor blocker (ARB).

of calf hypertrophy on the one hand and MRC sum score $(p=0.040)$ and FVC $(p=0.007)$ on the other hand. This suggests that calf hypertrophy was observed most often in patients in earlier disease stages. Two patients had mild scoliosis, not requiring treatment $(14 \%, 2 / 14)$. Eleven patients (48\%) had contractures in at least one location: nine patients in the ankles, six in the knees, three in the hips and three in the elbows (Table 1). In total, 14 patients were wheelchairbound $(61 \%)$, with a median age at loss of ambulation of 30 years (range 12-60y). Two of these patients had died, one of cardiac arrest (P13, aged 78y) and one of unknown cause (P16, aged 48y). Four patients required positive airway pressure treatment $(17 \%)$ : two were treated with non-invasive ventilation (NIV, one at night, one continuously) because of restrictive lung disease and two with continuous positive airway pressure therapy (CPAP) for obstructive sleep apnoea syndrome (OSAS). One additional patient was referred for a polysomnography and was diagnosed with mild OSAS, not requiring treatment $(\mathrm{P} 21)$. The same referral criteria for the evaluation of the need for NIV/CPAP were employed in both centres. During follow-up consultations, it was standard practice to inquire for clinical symptoms such as fatigue, dyspnoea, orthopnoea, matinal headache and monitor the FVC with regular pulmonary function tests [12]. Patients with OSAS had an average BMI of $30.3 \mathrm{~kg} / \mathrm{m}^{2}(\mathrm{SD} \pm 5.23)$, compared to $26.6 \mathrm{~kg} / \mathrm{m}^{2}$ ( $\left.\mathrm{SD} \pm 4.12\right)$ in the others $(p=0.264)$. Five patients $(22 \%)$ were active or past smokers and are indicated with an asterisk in Table 1.

Ten patients suffered from cardiomyopathy (43\%), of which nine had a dilated cardiomyopathy The other patient had a mild hypertrophic cardiomyopathy due to arterial hypertension. Six out of ten patients received treatment with Angiotensin Converting Enzyme (ACE)inhibitors (or Angiotensin II receptor blockers when indicated), complemented with beta blockers in five of them and additional diuretics in one (Table 2). One patient required a heart transplantation at the age of 25 years (P14, Tables 1 and 2), which was followed by lifelong immunosuppressive treatment with
Table 3

Estimates and p-values of pulmonary function test parameters vs. ageing.

\begin{tabular}{lll}
\hline Parameter & estimate & p-value \\
\hline FVC\%pred & -0.996 & 0.004 \\
FVC & -0.068 & $<0.001$ \\
FEV1\%pred & -1.378 & $<0.001$ \\
FEV1 & -0.072 & $<0.001$ \\
PEF & -0.102 & $<0.001$ \\
\hline
\end{tabular}

Forced Vital Capacity percentage of predicted (FVC\%pred), Forced Expiratory Volume in $1 \mathrm{~s}$ percentage of predicted (FEV1\%pred), Peak Expiratory Flow (PEF). Bold values are significant $(\alpha<0.05)$.

methylprednisolone, azathioprine and cyclosporine. Six patients showed cardiac conduction abnormalities (27\%, 6/22): right bundle branch block in two, a first-degree atrioventricular block in one and complete atrioventricular block in three patients requiring pacemaker implantation. All but one of these patients were treated with beta blockers (Table 2). The 23 unrelated patients harboured 14 different genetic defects in the $D M D$-gene. Six patients $(26 \%)$ presented a positive family history for BMD. Three patients carried an out of frame mutation and all of them had lost ambulation, of which one already at the age of 12 years (this patient also required NIV, P19), and the other two at the ages of 23 and 60 years. All three patients were currently aged well into their fifth or sixth decades corresponding to a BMD phenotype.

\subsection{The rate of pulmonary function decline and patient features affecting this deterioration}

We measured an annual decline of $1.00 \%$ of $\mathrm{FVC} \%$ pred $(p=0.004$, Fig. 1$)$, corresponding to a loss of $0.07 \mathrm{~L} \mathrm{FVC}$ per year in this BMD patient cohort $(p<0.001$, Table 3$)$. Other spirometry parameters also declined significantly each year: FEV1\%pred declined with $1.38 \%$ per year and FEV1 with $0.07 \mathrm{~L}(p<0.001$ for both). PEF declined with $0.10 \mathrm{~L}$ per year $(p<0.001)$, but PEF percent predicted data were missing. 


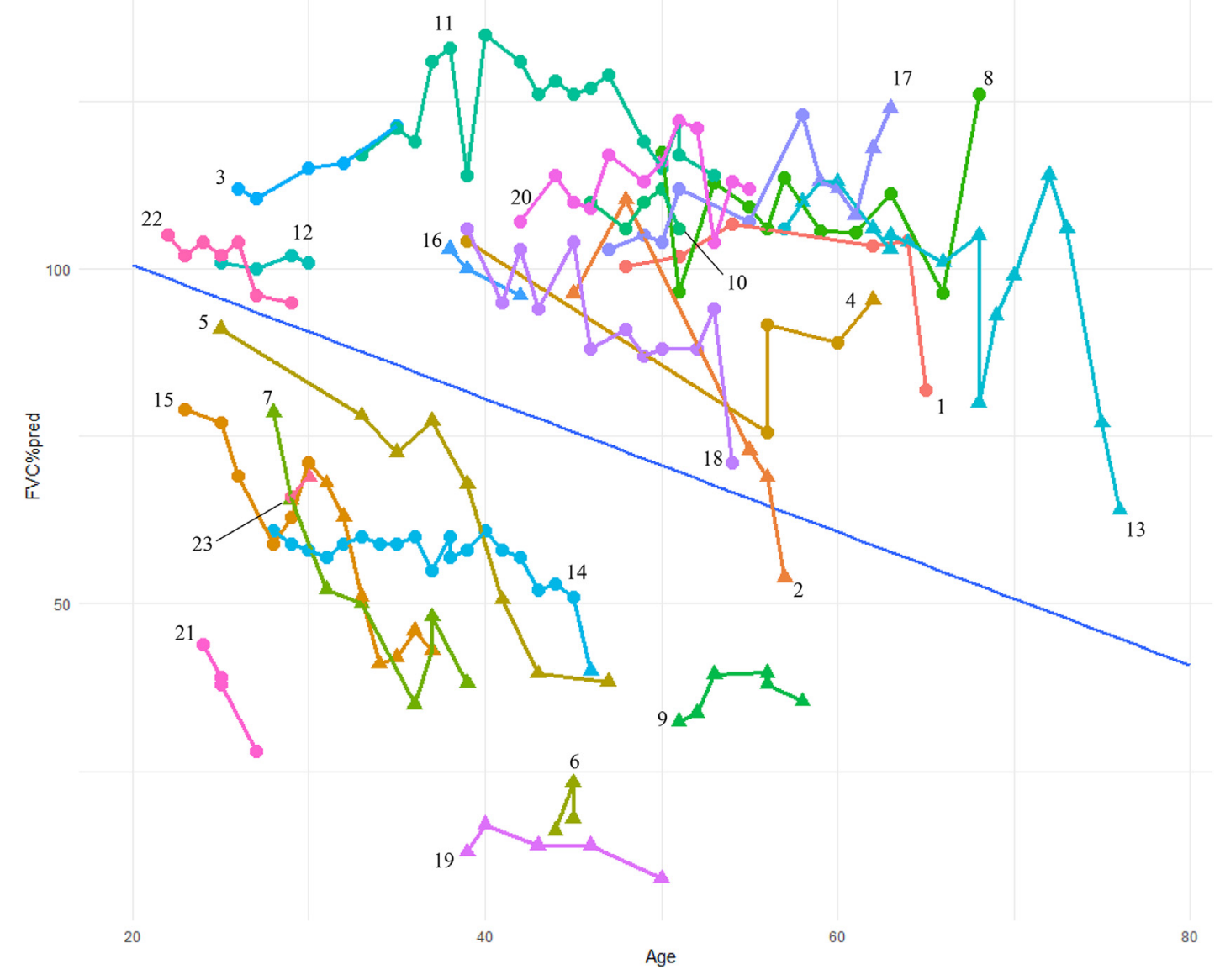

Fig. 1. FVC\%pred decline over time in our cohort of adult BMD patients.

Note two different overall trends in FVC percentage predicted (FVC\%pred): one group of patients experienced a rapid and early deterioration of FVC\%pred (below blue prediction line) and the other patient group presented only a mild deterioration (above prediction line). This largely corresponds to ambulatory status, with a loss of ambulation coinciding with a significantly more rapid FVC\%pred deterioration. Below the line, only P21 had not lost ambulation, but he became wheelchair-bound in the year following his last spirometry. Patients are colour-coded, and their number is annotated along their respective data points. Circles represent FVC\%pred measurements at which the patient was still ambulatory, triangles represent non-ambulatory patients. The blue prediction line depicts the annual decline of FVC\% \%red with $1.00 \%$ and is the result of the linear mixed model reported in more detail in Supplementary Table 1.

Table 4

Correlation of clinical characteristics with FVC over time.

\begin{tabular}{lll}
\hline Parameter & estimate & $p$-value \\
\hline Age & -0.032 & 0.015 \\
Years since loss of ambulation*BMI & -0.003 & 0.015 \\
BMI & -0.016 & 0.344 \\
Mutation frame & 0.425 & 0.595 \\
Age at symptom onset & 0.026 & 0.356 \\
\hline
\end{tabular}

Forced vital capacity (FVC), Body Mass Index (BMI). Bold values are significant $(\alpha<0.05)$, the asterisk $\left(^{*}\right)$ signifies an interaction term. The interaction should be interpreted as $-0.003 \mathrm{x}$ Years since loss of ambulation $x$ BMI. For example: a patient that lost ambulation 10 years ago and has a BMI of $25 \mathrm{~kg} / \mathrm{m}^{2}$ is predicted to have a $0.75 \mathrm{~L}$ lower FVC than a similar ambulatory patient.

Next, we evaluated whether patient characteristics such as BMI, genetic defect (in-frame vs. out of frame mutation), age at symptom onset and ambulatory status correlated with the rate of decline of FVC values over time (Table 4). The rate of FVC decline increased significantly with each year since loss of ambulation, and this effect was dependent of BMI (as signified by the interaction term in Table 4). Indeed, although all adult BMD patients in this study experienced a significant annual decline of FVC, the non-ambulatory patients declined significantly more rapidly $(p=0.015)$. Fig. 1 shows two overall trends in the data of FVC\%pred over age: a group of patients above the blue prediction line generally experienced a mild decline in FVC\%pred over time and patients below this line deteriorated more rapidly. Most patients above the prediction line were still ambulatory, whereas all patients below the prediction line lost ambulation (except for P21, but this patient also became wheelchair-bound in the year following his last spirometry).

The interaction between loss of ambulation and BMI manifests as a larger acceleration of annual FVC decline after loss of ambulation for patients with an increasing BMI. In contrast, BMI was not significantly correlated with FVC decline in ambulatory patients. In a post-hoc analysis, we evaluated whether wheelchair-bound patients experienced an increase in BMI due to sedentarism. However, we did 
Table 5

Estimates and p-values of clinical and cardiological parameters vs. FVC.

\begin{tabular}{lll}
\hline Parameter & estimate & $p$-value \\
\hline MRC sum score & 0.077 & 0.003 \\
Deltoid paresis & -0.230 & 0.782 \\
Limb contractures & 0.308 & 0.623 \\
LVEF & $<0.001$ & 0.984 \\
\hline
\end{tabular}

Forced vital capacity (FVC), Medical Research Council sum score (MRC sum score), left ventricular ejection fraction (LVEF). Bold values are significant $(\alpha<0.05)$.

not observe a difference in BMI based on ambulatory status $(p=0.297)$. All adult BMD patients experienced a slight increase in BMI with ageing $\left(+0.19 \mathrm{~kg} / \mathrm{m}^{2}\right.$ per year, $p<0.001$ ), irrespective of their ambulatory status.

Finally, patients with a higher age at symptom onset or an in-frame mutation (vs. out of frame) both showed a trend towards slower FVC decline, but these effects failed to reach significance.

\subsection{Correlation of FVC with the clinical neurological examination and LVEF}

Next, we analysed if the clinical neurological examination at our patients' last visit was correlated to their FVC at that time (Table 5). Data were corrected for patients' age. Only the MRC sum score correlated with FVC values, with a decline in skeletal muscle force correlating with lower FVC values $(p=0.003)$. For every point lost on the MRC sum score, $0.08 \mathrm{~L} \mathrm{FVC}$ was lost, indicating that a loss of 12.5 points on the MRC sum score was associated with a loss of $1 \mathrm{~L}$ FVC. We did not find a correlation between FVC and other parameters of the clinical neurological examination such as proximal arm weakness, particularly deltoid muscle paresis, or limb contractures (in at least 1 limb). There was no correlation between LVEF and FVC ( $p=0.984)$.

\section{Discussion}

Our study in a cohort of adult BMD patients revealed an annual decline of respiratory function of $1.00 \%$ of FVC\%pred. Furthermore, all evaluated parameters of the pulmonary function tests deteriorated significantly each year, including FVC, FEV1\%pred, FEV1 and PEF. Loss of ambulation significantly increased the annual rate of FVC decline and was influenced by patient's BMI. Indeed, an increase in BMI in wheelchair-bound patients was correlated with an accelerated FVC decline, whereas in ambulatory patients BMI and FVC were not significantly correlated. A decline in MRC sum score was significantly correlated with a decline in FVC, indicating a relation between skeletal and respiratory muscle function. The patient group in this study was comparable to the usual phenotype of BMD patients, with a similar average age at symptom onset and loss of ambulation, as well as similar rates of dilated cardiomyopathy $[5]$.

\subsection{Respiratory function decline in adult BMD patients}

We studied for the first time the evolution of respiratory function in BMD patients over time. In analogy, an annual decline in FVC\% \%red of around 5\% from the age of 12 years (which coincides with loss of ambulation) has been shown previously in DMD patients [13]. It is not surprising that the annual rate of respiratory decline we observed in this BMD cohort was much slower $(1 \%)$ than in DMD patients $(5 \%)$, since the BMD clinical phenotype is usually milder.

Interestingly, respiratory decline in this BMD population was mainly driven by non-ambulatory patients. Indeed, patients that remained ambulatory showed a trend towards FVC decline over time that was statistically significant, but had a minimal effect size (Fig. 1, Table 4). Adult ambulatory BMD patients in this study only lost $0.03 \mathrm{~L} \mathrm{FVC} \mathrm{per} \mathrm{year} \mathrm{and}$ would thus only experience a decline of $1 \mathrm{~L} \mathrm{FVC} \mathrm{after} \mathrm{more}$ than 33 years. Conversely, patients with a BMI of $25 \mathrm{~kg} / \mathrm{m}^{2}$ experienced a more than threefold increase in the rate of FVC decline after loss of ambulation, and patients with a higher BMI deteriorated even more rapidly.

We established that a significant respiratory decline can be expected in adult BMD patients, predominantly after loss of ambulation. An enhanced vigilance for respiratory complications is warranted in this subgroup.

In contrast to DMD, where scoliosis often develops rapidly after loss of ambulation during childhood, which adds to increased respiratory comorbidity in DMD, only two adult BMD patients had a mild scoliosis in this study. Although scoliosis is indeed less common in BMD in comparison with DMD, clinical and radiographic monitoring for this complication is warranted and referral for spinal fusion should be made when necessary [14].

The association between BMI and FVC in wheelchairbound, but not ambulatory patients, was another interesting finding of this study. One could hypothesize that wheelchairbound patients experience an increase in BMI due to sedentarism, and consequently loss of ambulation is the only driving factor for a more rapid FVC decline. However, in this study no difference in BMI with respect to ambulation status was observed. Based on these results, we would recommend increased attention for weight gain and overweight in BMD patients in clinical practice, especially after loss of ambulation. Dietary advice guidelines already exist for DMD patients [15]. Furthermore, patient organisations (such as the Muscular Dystrophy Association) offer advice on low caloric diets for obese BMD patients, although these are founded on expert opinion rather than clinical trials. The decrease of caloric intake is indeed the most important tool to facilitate weight loss after losing ambulation because of the decrease in mobility. A multidisciplinary approach including a dietician, with care for a healthy and balanced diet, is certainly recommended to avoid protein deficiency and malnutrition in BMD patients, in analogy with recommendations in DMD [16]. Cardiovascular health arguments are often proposed as the main reason for a low caloric diet, but our study suggests that a diet is also important to maintain a good pulmonary 
function in non-ambulatory BMD patients. Our results are not surprising, considering that other studies have shown that overweight is correlated with a lower total lung capacity in Myotonic Dystrophy type 1 patients, as well as a decrease in FVC and other respiratory parameters in healthy individuals $[17,18]$. Regarding the prevalence of OSAS in this study $(3 / 23$ or $13 \%$ ), we conclude that this is comparable to the general male population. The prevalence of OSAS in males in the general population is estimated at $3-7 \%$, but overweight is known to increase this risk up to fourfold, and the majority of patients in this study were indeed overweight (BMI > 25 in $70 \%)[19,20]$.

Next, we confirmed that a routine clinical examination with manual skeletal muscle force grading using the MRC sum score is relevant in the follow-up of adult BMD patients, since a decline of this score is correlated with a worsening of respiratory function. In contrast, we did not observe such a relation between FVC and cardiac muscle function, as measured by the LVEF. Although these results are not surprising since respiratory muscles are also skeletal muscles, it should be noted that the time of occurrence of decline in FVC and increase in muscle weakness is different, as clinically relevant respiratory decline only took place after loss of ambulation. We also did not find a significant correlation between FVC and presence of deltoid muscle paresis or contractures. Deltoid muscle paresis (graded on the MRC score) was specifically included in this assessment, because it is known to serve as a warning sign for impending respiratory failure in subacute skeletal muscular paresis caused by neuromuscular disorders [21]. However, in the BMD patients, that all experienced chronic respiratory decline, no such relation was observed. A correlation between pulmonary function tests and the Brooke score, an outcome measure based on muscle function in the upper limbs, was recently shown in DMD patients [22]. However, no such data were available for analysis in this retrospective study. Assessment of additional outcome measures based on muscle function, that are known to be relevant in DMD, such as the six-minute walking distance (6MWD) or the North Star Ambulatory Assessment (NSAA) are warranted in future prospective studies in adult BMD patients.

No in-depth genotype-based sub-analysis was performed in this study, given the presence of 14 different mutations in our population of 23 patients. However, we studied differences between in vs. out of frame mutations and showed a slower pulmonary function decline in patients with inframe mutations, although this was not significant. This was probably due to the fact that only $3 / 23$ patients had an out of frame mutation.

Finally, this study confirmed that FVC could potentially serve as an outcome measure in adult BMD patients because of its significant annual decline in this population. This is in line with previous findings in DMD patients and other muscle diseases such as late-onset Pompe disease (LOPD), where FVC has been proposed as a valid outcome measure $[7,21]$. However, as we have demonstrated that the annual decline in FVC in ambulatory adult BMD patients is very slow (although statistically significant), its relevance as an outcome measure appears to be limited to a non-ambulatory BMD population. This restricts the use of FVC as an outcome measure in clinical trials, as these frequently tend to focus on earlier disease stages (before loss of ambulation). Nevertheless, pulmonary function tests are relatively cheap, easily performed and widely available and the implications for respiratory follow-up of non-ambulatory adult BMD patients in clinical practice remain highly relevant.

\subsection{Cardiac function in adult BMD patients}

In this study, annual screening of BMD patients with an electrocardiogram (ECG) and echocardiography was part of the standard of care in both participating neuromuscular centres, in compliance with the guidelines from the European Neuromuscular Centre (ENMC) and American Heart Association (AHA) [23,24]. The importance of rigorous cardiac follow-up derives from the high frequency of cardiac complications even in subclinical or mild BMD patients and from the fact that severe left ventricular dysfunction is a negative prognostic factor [25-27].

The frequency of cardiac complications such as cardiomyopathy and/or arrhythmias was 57\% (13/23) in this adult BMD population and thereby comparable to the often cited $60-75 \%$ in the literature [5,25]. The majority of the patients in this study were treated in accordance with the consensus guidelines, that recommend initiation of ACE-inhibitors with or without beta blockers upon diagnosis of cardiomyopathy (most often defined as LVEF $<55 \%$ ) [28].

In analogy with findings in DMD children, the preventive use of perindopril or the association of eplerenone to ACEinhibitors might also be beneficial to the cardiac function in BMD patients [29,30]. Both options were not performed in the current BMD population, with the exception of one patient (P5, Table 2), that received ACE-I because of a drop to a borderline-low LVEF without clear arguments for a dilated cardiomyopathy. Although the field of therapeutic development is certainly evolving more rapidly in DMD as opposed to BMD, novel approaches in BMD are also noteworthy. Therapeutic options such as phosphodiesterase type 5 (PDE5)-inhibitors to increase the nitric oxide (NO) signalling pathway have been described in the literature with mixed results and might also be primarily useful in earlier disease stages [31]. Furthermore, although endurance training was not found to alter cardiac pump function in adult BMD patients, an increase of maximum volume of oxygen uptake (VO2max) and skeletal muscle strength was observed, implicating the beneficial effect of active rehabilitation in these patients [32].

In this study, four of the 13 patients developing cardiac complications were currently still ambulatory, three had lost ambulation prior to the development of cardiac complications and three had only lost ambulation afterwards. Of the three other non-ambulatory patients the temporal relation between the development of cardiac complications and loss 
of ambulation was not discernible with certainty from the patients' medical files. The lack of a clear association between loss of ambulation and cardiac complications is in agreement with the conclusion from previous studies that there is no direct relation between cardiac and skeletal muscle disease in BMD patients [5].

\subsection{Limitations of the study}

The most important limitations of this study are its retrospective nature and relatively small sample size. Although it is well known that BMD is a clinically heterogeneous disorder, patients from the milder end of the disease spectrum that only display symptoms such as fatigue, muscle cramps or hyperCKemia are not represented in this study. However, the selection of only a subset of the disease spectrum can sometimes be preferable to draw conclusions, as was recently shown in another group of muscular dystrophies, i.e. sarcoglycanopathies [33]. However, in order to increase the understanding of disease progression for the entire spectrum of BMD patients and develop efficacious multimodal interventions, larger prospective natural history studies are required in the future.

\section{Conclusions}

We conclude that adult BMD patients experience a significant but mild respiratory decline. However, this decline is significantly more rapid and clinically relevant after loss of ambulation, which warrants a more vigilant follow-up of respiratory function in this subgroup. Despite its significant annual decline, FVC has rather limited potential as an outcome measure in clinical trials in adult BMD patients. BMI deserves special attention in clinical practice since overweight is correlated with a significant deterioration of FVC in nonambulatory BMD patients. There is a significant link between a decline in FVC and MRC sum score, but this is not observed with LVEF.

\section{Ethics declarations}

This retrospective study was approved by the respective local Ethics Committees of the two participating centres.

\section{Funding}

This research did not receive any specific grant from funding agencies in the public, commercial, or not-for-profit sectors.

\section{Disclosures}

None.

\section{Declaration of Competing Interest}

KGC is Chairholder of the Emil von Behring Chair for Neuromuscular and Neurodegenerative Disorders by CSL
Behring. KGC and several authors of this publication are member of the European Reference Network for Rare Neuromuscular Diseases (ERN EURO-NMD) and of the European Reference Network for Rare Neurological Diseases (ERN-RND). The authors have no competing interests.

\section{Data for reference}

The supporting datasets analysed during the current study are available from the corresponding author on reasonable request.

\section{Acknowledgements}

All authors were involved in the data collection and critical revision of the article for the intellectual content and final approval of the version intended to be published. BDW and KGC performed statistical analyses and analysed and interpreted the patient data.

\section{Supplementary materials}

Supplementary material associated with this article can be found, in the online version, at doi:10.1016/j.nmd.2020.12. 010 .

\section{References}

[1] Gangopadhyay SB, Sherratt TG, Heckmatt JZ, et al. Dystrophin in frameshift deletion patients with Becker muscular dystrophy. Am J Hum Genet 1992;51(3):562-70.

[2] Bushby KMD, Thambyayah M, Gardner-Medwin D. Prevalence and incidence of Becker muscular dystrophy. Lancet 1991;337(8748):1022-4.

[3] Bushby KMD, Gardner-Medwin D. The clinical, genetic and dystrophin characteristics of Becker muscular dystrophy - I. Natural history. J Neurol 1993;240(2):98-104.

[4] Birnkrant DJ, Ashwath ML, Noritz GH, et al. Cardiac and pulmonary function variability in Duchenne/Becker muscular dystrophy: an initial report. J Child Neurol 2010;25(9):1110-15.

[5] Ho R, Nguyen M-L, Mather P. Cardiomyopathy in Becker muscular dystrophy: overview. World J Cardiol 2016;8(6):356.

[6] PB S. Emerging strategies in the treatment of Duchenne muscular dystrophy. Neurotherapeutics 2018;15(4):840-8.

[7] Phillips MF, Quinlivan RCM, Edwards RHT, Calverley PMA. Changes in spirometry over time as a prognostic marker in patients with Duchenne muscular dystrophy. Am J Respir Crit Care Med 2002;164(12):2191-4.

[8] Khirani S, Ramirez A, Aubertin G, et al. Respiratory muscle decline in Duchenne muscular dystrophy. Pediatr Pulmonol 2014;49(5):473-81.

[9] Quanjer PH, Stanojevic S, Cole TJ, et al. Multi-ethnic reference values for spirometry for the 3-95-yr age range: the global lung function 2012 equations. Eur Respir J 2012;40(6):1324-43.

[10] Kleyweg RP, Van Der Meché FGA, Schmitz PIM. Interobserver agreement in the assessment of muscle strength and functional abilities in Guillain-Barré syndrome. Muscle Nerve 1991;14(11):1103-9.

[11] Ponikowski P, Voors AA, Anker SD, et al. 2016 ESC Guidelines for the diagnosis and treatment of acute and chronic heart failure: the Task Force for the diagnosis and treatment of acute and chronic heart failure of the European Society of Cardiology (ESC). Developed with the special contribution of the Heart Failure Association (HFA) of the ESC. Eur J Heart Fail 2016;18(8):891-975. 
[12] Priou P, Trzepizur W, Meslier N, Gagnadoux F. Update on the respiratory management of patients with chronic neuromuscular disease. Rev Pneumol Clin 2017;73(6):316-22.

[13] Kinane TB, Mayer OH, Duda PW, Lowes LP, Moody SL, Mendell JR. Long-term pulmonary function in Duchenne muscular dystrophy: comparison of Eteplirsen-treated patients to natural history. J Neuromuscul Dis 2018;5(1):47-58.

[14] Archer JE, Gardner AC, Roper HP, Chikermane AA, Tatman AJ. Duchenne muscular dystrophy: the management of scoliosis. J Spine Surg 2016;2(3):185-94.

[15] Davis J, Samuels E, Mullins L. Nutrition considerations in Duchenne muscular dystrophy. Nutr Clin Pract 2015;30(4):511-21.

[16] Salera S, Menni F, Moggio M, Guez S, Sciacco M, Esposito S. Nutritional challenges in duchenne muscular dystrophy. Nutrients 2017;9(6).

[17] Seijger CGW, Drost G, Posma JM, van Engelen BGM, Heijdra YF. Overweight is an independent risk factor for reduced lung volumes in myotonic dystrophy type 1. PLoS One 2016;11(3).

[18] Melo LC, da Silva MAM, do Nascimento Calles AC. Obesity and lung function: a systematic review. Einstein 2014;12(1):120-5.

[19] Garvey JF, Pengo MF, Drakatos P, Kent BD. Epidemiological aspects of obstructive sleep apnea. J Thorac Dis 2015;7(5):920-9. doi:10.3978/ j.issn.2072-1439.2015.04.52.

[20] Young T, Palta M, Dempsey J, Skatrud J, Weber S, Badr S. The occurrence of sleep-disordered breathing among middle-aged adults. N Engl J Med 1993;328(17):1230-5.

[21] Ishikawa Y. Neuromuscular disease and respiratory organ. Respir Circ 2009;57(3):285-90.

[22] Mayer OH. Pulmonary function and clinical correlation in DMD. Paediatr Respir Rev 2019;30:13-15.

[23] Feingold B, Mahle WT, Auerbach S, et al. Management of cardiac involvement associated with neuromuscular diseases: a scientific statement from the American Heart Association. Circulation 2017;136(13):e200-31.
[24] Bushby K, Muntoni F, Bourke JP. 107th ENMC International Workshop: the management of cardiac involvement in muscular dystrophy and myotonic dystrophy. 7th-9th June 2002, Naarden, the Netherlands. Neuromuscul Disord 2003;13(2):166-72.

[25] Melacini P, Fanin M, Danieli GA, et al. Cardiac involvement in Becker muscular dystrophy. J Am Coll Cardiol 1993;22(7):1927-34.

[26] Melacini P, Fanin M, Danieli GA, et al. Myocardial involvement is very frequent among patients affected with subclinical Becker's muscular dystrophy. Circulation 1996;94(12):3168-75.

[27] Angelini C, Fanin M, Freda MP, et al. Prognostic factors in mild dystrophinopathies. J Neurol Sci 1996;142(1-2):70-8.

[28] Angelini C, Marozzo R, Pegoraro V. Current and emerging therapies in Becker muscular dystrophy (BMD). Acta Myol 2019;38(3):172-9.

[29] Duboc D, Meune C, Lerebours G, Devaux JY, Vaksmann G, Bécane HM. Effect of perindopril on the onset and progression of left ventricular dysfunction in Duchenne muscular dystrophy. J Am Coll Cardiol 2005;45(6):855-7.

[30] Raman SV, Hor KN, Mazur W, et al. Eplerenone for early cardiomyopathy in Duchenne muscular dystrophy: a randomised, double-blind, placebo-controlled trial. Lancet Neurol 2015;14(2):153-61.

[31] Dombernowsky NW, Ölmestig JNE, Witting N, Kruuse C. Role of neuronal nitric oxide synthase (nNOS) in Duchenne and Becker muscular dystrophies - still a possible treatment modality? Neuromuscul Disord 2018;28(11):914-26.

[32] Sveen ML, Jeppesen TD, Hauerslev S, Køber L, Krag TO, Vissing J. Endurance training improves fitness and strength in patients with Becker muscular dystrophy. Brain 2008;131(11):2824-31.

[33] Alonso-Pérez J, González-Quereda L, Bello L, et al. New genotype-phenotype correlations in a large European cohort of patients with sarcoglycanopathy. Brain 2020;143(9):2696-708. 